\title{
The Effect of Collisions on Wave-Breaking in a Cold Plasma
}

\author{
A. Schmitt \\ Department of Nuclear Engineering, The University of Michigan, Ann Arbor, MI 48109, \\ USA \\ R. S. B. Ong \\ Department of Aerospace Engineering, The University of Michigan, Ann Arbor, MI 48109, \\ USA
}

Received 15 February 1980/Accepted 19 March 1980

\begin{abstract}
The effect of electron collisions on reducing the wave-breaking amplitude of resonantly-driven electrostatic fields in a cold plasma is investigated. By means of a simple theory collisions are shown to compete with wave-breaking in dissipating wave energy.
\end{abstract}

PACS: $52.35,52.50$

Resonance absorption is one of the effective ways in which electromagnetic waves can deposit large amounts of energy in a plasma. In the process, the incident electromagnetic wave drives a Langmuir wave in the neighborhood of the plasma critical density, i.e., the region where the local electron plasma frequency equals the incident wave frequency. The resulting electrostatic wave grows and nonlinear wave-breaking can occur with the consequent production of energetic (suprathermal) electrons [1]. This last effect is undesirable in laser fusion applications, since the hot electrons can pre-heat the pellet core prior to compression and thereby adversely affect neutron yield.

It is known that ion dynamics and thermal effects modify the wave breaking amplitude $[2,3]$. They reduce the role of the wave-breaking process of energy dissipation by providing competing modes of energy release. It is shown in this paper that electron-ion collisions produce a similar effect. Collisions transfer the energy of the oscillating electrons to the ions, which are modeled here as a background momentum sink. This process reduces the level of hot electron production and localizes the absorbed energy.

\section{Theory}

Consider an electromagnetic wave incident upon a plasma with a linear density profile. Let $E_{d}$ be the component of the incident wave electric field parallel to the density gradient. A Lagrangian system of coordinates will be used to describe the nonlinear electron plasma oscillations under the influence of the driver field $E_{d}$ [4].

In the Lagrangian coordinate system, the position of each electron, $\delta$, is measured relative to its individual initial position $x_{0}$. In contrast, the customary Eulerian coordinates measure the position of the electron, $x(t)$, relative to a single fixed point $(x=0)$. The two coordinate systems are related through the simple relation

$\delta\left(x_{0}, t\right)=x(t)-x_{0}$.

From the equation of motion of the electrons (including the collision term) and Poisson's equation, it is found that the electron displacement $\delta$ satisfies

$\ddot{\delta}+v \dot{\delta}+\omega_{p e}^{2}\left(x_{0}\right) \delta=\varepsilon \sin t$,

where $\varepsilon=e E_{d} / m_{e} \omega_{d}^{2} L ; \omega_{d}$ is the frequency of the incident electromagnetic wave; $L$ is a characteristic distance, e.g., the density gradient scale length. All distances and times in (2) are normalized with respect to $L$ and $\omega_{d}^{-1}$, respectively. Thus, (2) is an equation in dimensionless form. The effect of collisions is taken into account by the second term in (2) where $v$ is proportional to the electron-ion collision frequency. It has been implicitly assumed in (2) that $\delta \ll 1$. 


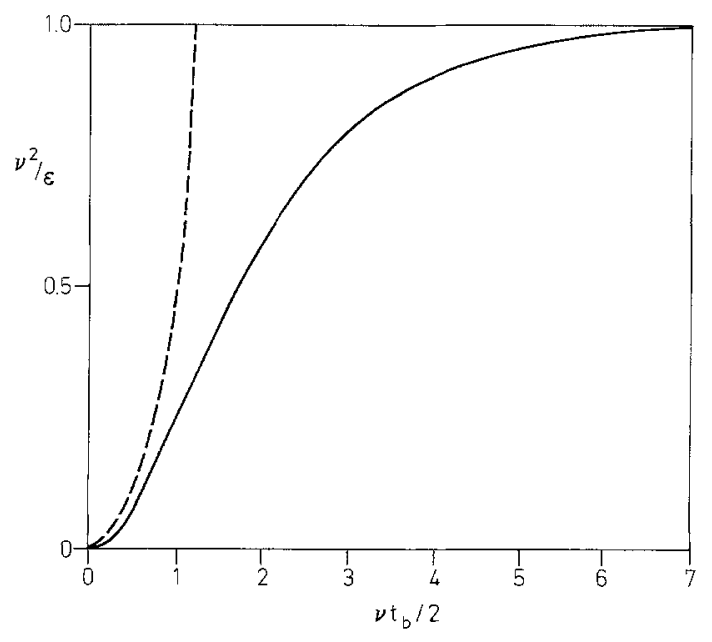

Fig. 1. The right hand side of the relation $v^{2} / \varepsilon=1-\exp \left(-v t_{b} / 2\right)\left(1+v t_{b} / 2\right)$ plotted as a function of the wavebreaking time. The dotted curve shows the wave-breaking time in the absence of collisions

The solution of (2) is given by

$$
\begin{aligned}
\delta(t)= & {\left[e / \cos \gamma\left(\omega_{p}^{2}-1+v \tan \gamma\right)\right] } \\
& \cdot\left\{\operatorname { e x p } ( - v t / 2 ) \left[\sin \gamma \cos \left[\left(\omega_{p}^{2}-\frac{1}{4} v^{2}\right)^{1 / 2} t\right]\right.\right. \\
& \left.+\left(\frac{1}{2} v \sin \gamma-\cos \gamma\right) \sin \left[\left(\omega_{p}^{2}-\frac{1}{4} v^{2}\right)^{1 / 2}\right] /\left(\omega_{p}^{2}-\frac{1}{4} v^{2}\right)^{1 / 2}\right] \\
& +\sin (t-\gamma)\},
\end{aligned}
$$

where $\gamma \equiv \tan ^{-1}\left(v / \omega_{p}^{2}-1\right)$. Let us now consider the behavior of $\delta(t)$ at the resonance region where the electrostatic wave grows most rapidly. We take the limit as $\omega_{p} \rightarrow 1$ and let $v^{2} \ll \omega_{p}^{2}$. The result is

$\delta(t)=(\varepsilon / v)\left[\exp (-v t / 2)\left(\cos t+\frac{1}{2} v \sin t\right)-\cos t\right]$.

Note the limiting effect that the $\exp (-v t / 2)$ factor has on the wave amplitude. Clearly, for sufficiently large values of $v$ the electrostatic wave is drastically modified. It is also to be observed that in the limit $v \rightarrow 0$ the collisionless result of Koch and Albritton is recovered.

In order to examine the effect of the collision frequency in more detail we consider the time $t_{b}$ at which the wave breaks. This condition occurs when $\partial \delta / \partial x_{0}=-1$, i.e., when the electron paths cross one another. We now apply this condition to (4) and find that $t_{b}$ satisfies the relation

$v^{2} / \varepsilon=1-\exp \left(-v t_{b} / 2\right)\left(1+\frac{1}{2} v t_{b}\right)$.

This relation is shown graphically in Fig. 1. The important thing to note is that the right hand side is never larger than unity. Thus, if $v>\varepsilon^{1 / 2}$, the electrostatic wave cannot break. Instead of the wave to break and disperse its energy into hot electrons that stream out of the region, the electron-ion collisions localize the wave energy in the resonance region.

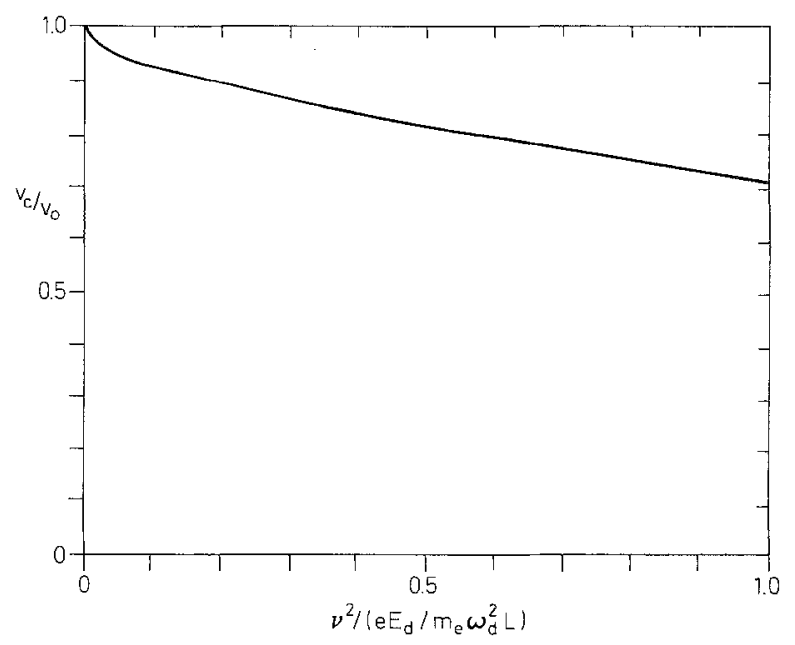

Fig. 2. The electron velocity at wave-breaking vs. the (scaled) collision frequency. $V_{0}=(2 \varepsilon)^{1 / 2}$ is the electron velocity at wavebreaking in the case of a collisionless plasma

Thus, to prevent wave-breaking the electron-ion collision frequency has to exceed $\left(e E_{d} / m_{e} \omega_{d}^{2} L\right)^{1 / 2}$. For lower values of $v$ the wave does break, but is now modified by collisions. Aside from the the much longer breaking time, the resulting electron velocity $V_{c}$ is also reduced. The electron velocity at wave-breaking $V_{c}$ is obtained by taking the time-derivative of $\delta$ in (4) and evaluating it at $t=t_{b}$. Figure 2 shows the electron velocity at wave-breaking as a function of the collision frequency. Thus collisions can significantly reduce the energy carried away by the hot electrons.

Finally, the limitations of this analysis need to be pointed out. With the longer wave-breaking times the effect of ion bunching becomes more important. However, this can be offset somewhat by consideration of ion collisions. Furthermore, as collisions transfer energy from the oscillating electrons to the ions, temperature effects would become significant. Finally, it is tacitly implied in the above analysis that $v t>1$, i.e., the electrons suffer at least one collision before the wave breaks.

Acknowledgments. This work was supported by Grant AFOSR-800029. Valuable discussions with Don Kania and David Voss are gratefully acknowledged. The authors also acknowledge the helpful comments of the Referee.

\section{References}

1. J.P.Freidberg, R.W.Mitchell, R.L.Morse, L.I.Rudsinski: Phys. Rev. Lett. 28, 795 (1972)

2. T.P.Coffey: Phys. Fluids 14, 1402 (1971)

3. P.Koch, J.Albritton: Phys. Rev. Lett. 32, 1420 (1974)

4. T. Speziale, P.J.Catto: Phys. Fluids 22, 681 (1979) 